\title{
MODELAGEM MATEMÁTICA DA CINÉTICA DE CLARIFICAÇÃO DO CALDO DE CANA MEDIANTE OZONIZAÇÃ̃O EM REATOR BATELADA
}

\author{
R. BERNARDI ${ }^{1}$, P.R. PARAÍSO ${ }^{2}$ e L. M. M. JORGE ${ }^{2}$ \\ ${ }^{1}$ Universidade Tecnológica Federal do Paraná, Departamento de Engenharia Eletrônica \\ ${ }^{2}$ Universidade Estadual de Maringá, Departamento de Engenharia Química \\ E-mail para contato: ricardobernardi@utfpr.edu.br \\ paulo@deq.uem.br \\ lmmj@deq.uem.br
}

\begin{abstract}
RESUMO -O modelo apresentado neste trabalho foi determinado através do balanço de massa dos pigmentos que dão cor ao caldo de cana tomando o reator como volume de controle. Sabendo-se que a cor do caldo pode ser considerada proporcional à absorbância da solução medida no comprimento deonda $\lambda=420 \eta \mathrm{m}$, o modelo resultou numa equação em função da absorbância e de três coeficientes. A validação do modelo foi obtida através de dados experimentais de absorbância, em reator batelada, para três temperaturas distintas: 10,30 e $50^{\circ} \mathrm{C}$. A determinação dos coeficientes para ajuste do modelo foi feita pelo método numérico, através de um script desenvolvido em MATLAB. A comparação dos dados experimentais com o modelo obteve bons resultados. Concluiu-se, com isso, que o modelo representa bem a cinética de clarificação do caldo de cana por ozonização em reator do tipo batelada.
\end{abstract}

\section{INTRODUÇÃO}

Alvo de disputas e conquistas durante séculos, a cana-de-açúcar encontrou lugar ideal no Brasil.Durante o império, estima-se que a cana-de-açúcar rendeu ao Brasil cinco vezes mais que todos os outros produtos agrícolas destinados à exportação. Atualmente o Brasil se mantém líder mundial na produção e exportação de açúcar sendo responsável por mais de $40 \%$ das exportações.

A produção do açúcar de alta qualidade, tipo exportação, ocorre mediante clarificação do caldo de cana. O processo de clarificação tradicionalmente utilizado pelas agroindústrias sucroalcooleiras é caracterizado por uma etapa conhecida por sulfitação, que libera resíduos de enxofre prejudiciais à saúde do ser humano e ao meio ambiente (SILVA, 2008).

O CodexAlimentarius, norma internacional de alimentos, produzida em conjunto pela FAO e OMS, admite um limite máximo de 15 miligramas de $\mathrm{SO}_{2}$ por quilograma de açúcar branco, para consumo direto, mas há movimentos internacionais no sentido de se reduzir esse limite a10mg/kg.A presença de resíduos de dióxido de enxofre restringe as exportações brasileiras de açúcar branco, eis que diversos países importadores não admitem a presença dessa substância nos alimentos que ali ingressam (RIBEIRO, 2009). 
Ao longo da história, vários processos alternativos foram estudados com objetivo de substituir a sulfitação no processo de clarificação do caldo de cana-de-açúcar. Atualmente podemos citar diversos trabalhos científicos publicados relacionados ao tema, tais como: bicarbonatação (ARAÚJO, 2007), carbonatação (HAMERSKI, 2009), filtração tangencial (ARMOA, 2012), uso de elétrons acelerados (LIMA, 2012) dentre outros.

Uma alternativabarata para a substituição do enxofre no processo de clarificação do caldo de cana-de-açúcar é a ozonização. O método consiste em injetar ozônio diretamente na tubulação de entrada da coluna ou torre de clareamento. Além de dispensar matérias primas, não requer grandes alterações na estrutura física presentes hoje nas usinas (SILTON, 2007).

Optou-se pelo estudo da clarificação de caldo de cana-de-açúcar por ozonização por acreditar-se que trata-se de uma alternativa promissora na substituição do processo de sulfitação. Além disso, ainda não existem publicações relevantes relacionadas a este tema. $\mathrm{O}$ modelo apresentado neste artigo poderá ser utilizado, ainda, em outras aplicações de clarificação mediante ozonização em reator do tipo batelada.

\section{LEVANTAMENTO DE DADOS DE EXPERIMENTAIS}

O levantamento de dados experimentais de absorbância em função do tempo é essencialpara a determinação dos coeficientes do modelo matemático proposto neste artigo. Para tanto, foram realizados ensaios de clarificação de caldo de cana-de-açúcarpara três temperaturas: 10,30 ou $50^{\circ} \mathrm{C}$. Umdiagrama do sistema de clarificação utilizado nos ensaios pode ser visto na Figura 1.

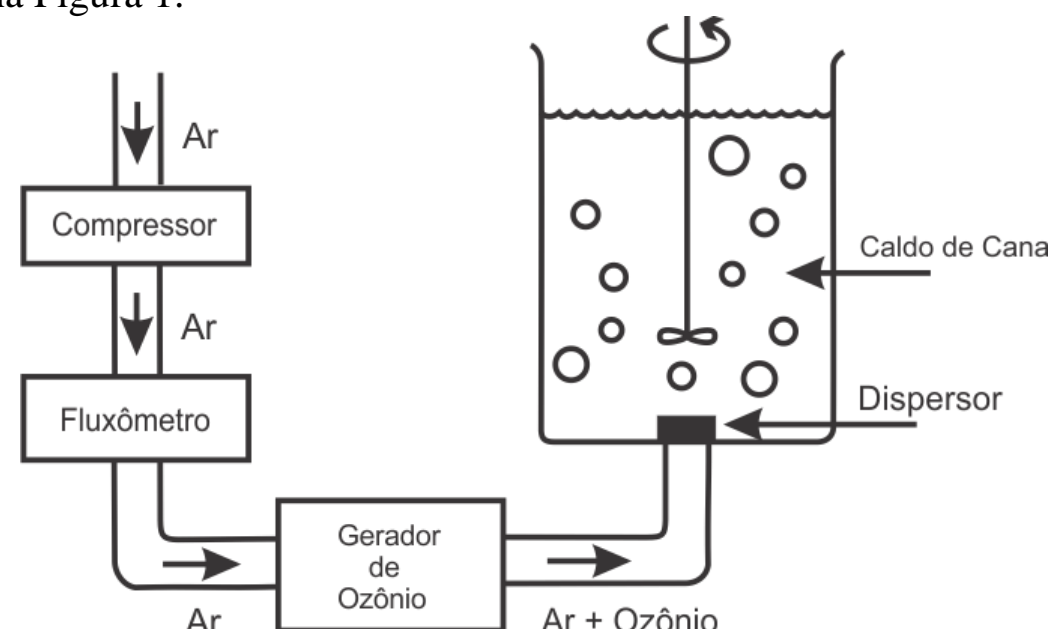

Figura 1 - Sistema de Clarificação

\subsection{MATERIAIS E MÉTODOS}

Primeiramente ajustou-se o gerador de ozônio (SCHIAVON, 2012) para operar com duas células em paralelo com vazão de ar ajustada, através de um fluxômetro, para $9 \mathrm{~L} / \mathrm{min}$ e tensão aplicada, em cada célula, de $60 \mathrm{~V}$. Em seguida, com o auxílio de um balão volumétrico de $200 \mathrm{ml}$ adicionou-se $200 \mathrm{~mL}$ de água destilada a uma proveta de $250 \mathrm{~mL}$. Na sequência, com uma pipeta de $50 \mathrm{~mL}$, adicionou-se $50 \mathrm{~mL}$ de caldo de cana-de-açúcar puro à mesma 
proveta de $250 \mathrm{~mL}$, formando uma solução de caldo de cana com concentração de $20 \%$. Transferiu-se então os $250 \mathrm{~mL}$ da solução para uma proveta de $1 \mathrm{~L}$ que foi colocada em banho termostático até atingir a temperatura desejada $\left(10,30\right.$ ou $\left.50{ }^{\circ} \mathrm{C}\right)$. Atingida a temperatura, o gerador de ozônio foi ligado por 15 segundos para estabilizar a geração de ozônio. Depois disso, as mangueiras do gerador foram inseridas dentro da proveta de $1 \mathrm{~L}$. Neste mesmo momento disparou-se o cronômetro. Decorrido o tempo determinado, desligou-se o gerador de ozônio eretirou-se as mangueiras da solução. Com o auxílio de uma pipeta, transferiu-se solução ozonizada suficiente para encher a cubeta utilizada para a medição da absorbância. A cubeta foi inserida no espectrofotômetro Hach, modelo DR 5000, ajustado para medir absorbância no comprimento de onda $\lambda=420 \eta \mathrm{m}$. O valor da absorbância foi medido e anotado. Cada ensaio foi realizado em duplicata para os tempos de ozonização de 2, 4, 6, 8 e 10 minutos.

\section{MODELO MATEMÁTICO}

Para o desenvolvimento do modelo matemático, admitiu-se que a reação é homogênea e dada pela equação1.

$$
\mathrm{P}+\mathrm{O}_{3} \rightarrow \mathrm{C}
$$

em que: $P=$ Solução pigmentada $C=$ Solução clarificada.

e considerou-se a taxa de reação dada pela equação 2. (FOGLER, 2008)

$$
-r_{p}=k_{p} C_{p}^{\alpha} C_{O_{3}}^{\beta}
$$

em que: $r_{p}=$ Taxa de reação de pigmentos; $k_{p}=$ Constante de velocidade de reação; $C_{p}=$ Concentração de pigmentos; $C_{O_{3}}=$ Concentração de ozônio; $\alpha=$ Ordem da reação de pigmentos; $\beta=$ Ordem da reação de ozônio.

O balanço de massa para os pigmentos no reator é dado pela equação 3 .

$$
A=E-S+G-C
$$

em que: $A=$ Taxa de massa de pigmentos acumulada no reator; $E=$ Taxa de massa de pigmentos que entra no reator; $S=$ Taxa de massa de pigmentos que sai do reator; $G=$ Taxa de massa de pigmentos que é gerada no reator; $C=$ Taxa de massa de pigmentos que é consumida no reator.

Sabendo que não entram $(E=0)$ ou saem $(S=0)$ pigmentos no reator e assumindo que nenhum pigmento é gerado $(\mathrm{G}=0)$ dentro do reator, o balanço de massa de pigmentos é agora representado pela equação 4 .

$A=-C$

Reescrevendo a equação 4 obtemos a equação 5. 
$\frac{d C_{p}}{d t}=r_{p}$

Substituindo a equação 2 em 5 obtém-se a equação 6 .

$\frac{d C_{p}}{d t}=-k_{p} C_{p}^{\alpha} C_{O_{3}}^{\beta}$

Considerando que a vazão de ozônio é alta suficiente para que a concentração de ozônio no reator mantenha a solução saturada, pode-se admitir que:

$C_{O_{3}}^{\beta}=k_{o}$

em que: $k_{o}=$ Concentração de ozônio constante.

Ainda, admitindo-se que a concentração de pigmentos é proporcional à absorbância nocomprimento de onda $\lambda=420 \eta \mathrm{m}$ (HUNTERLAB, 2008), tem-se que:

$C_{p}=k_{a} A_{\lambda}$

em que: $k_{a}=$ Constante de proporção entre os pigmentos e absorbância; $A_{\lambda}=$ Absorbância no comprimento de onda $\lambda=420 \eta \mathrm{m}$.

Substituindo as equações 7 e 8 na equação 6, tem-se:

$k_{a} \frac{d A_{\lambda}}{d t}=-k_{p} k_{o} k_{a}^{\alpha} A_{\lambda}^{\alpha}$

Dividindo a equação 9 por $k_{a}$, tem-se:

$\frac{d A_{\lambda}}{d t}=-k_{p} k_{o} k_{a}^{\alpha-1} A_{\lambda}^{\alpha}$

Aplicando a integral na equação 10 , tem-se:

$\int_{A_{0}}^{A} \frac{d A_{\lambda}}{d A_{\lambda}^{\alpha}}=\int_{t_{0}}^{t}-k_{p} k_{o} k_{a}^{\alpha-1} d t$

Resolvendo a integral, obtém-se:

$\frac{A^{1-\alpha}-A_{0}^{1-\alpha}}{1-\alpha}=-k_{p} k_{o} k_{a}^{\alpha-1}\left(t-t_{0}\right)$

Reescrevendo obtemos a equação modelo:

$A^{1-\alpha}=A_{0}^{1-\alpha}-k_{p} k_{o} k_{a}^{\alpha-1}(1-\alpha)\left(t-t_{0}\right)$ 
em que: $A=$ Absorbância em um dado tempo; $A_{0}=$ Absorbância inicial; $t=$ Final do intervalo de tempo; $t_{0}=$ Início do intervalo de tempo;

Os coeficientes da equação 13 foram obtidos por meio do ajuste não linear do modelo aos dados obtidos experimentalmente.Tal ajuste foi realizado através de um scriptdesenvolvido em MATLAB.

\section{RESULTADOS E DISCUSSÕES}

A Figura 2a mostra os pontos referentes aos dados de absorbâncias experimentais juntamente com as curvas ajustadas individualmente de acordo com a temperatura. Para cada temperatura obteve-se um conjunto diferente de coeficientes para a equação modelo. Portanto, obtendo um modelo diferente para cada temperatura estudada. Um gráfico das absorbâncias experimentais $v s$ absorbâncias calculadas pelostrês modelos ajustados individualmente pode ser visto na Figura $2 b$.

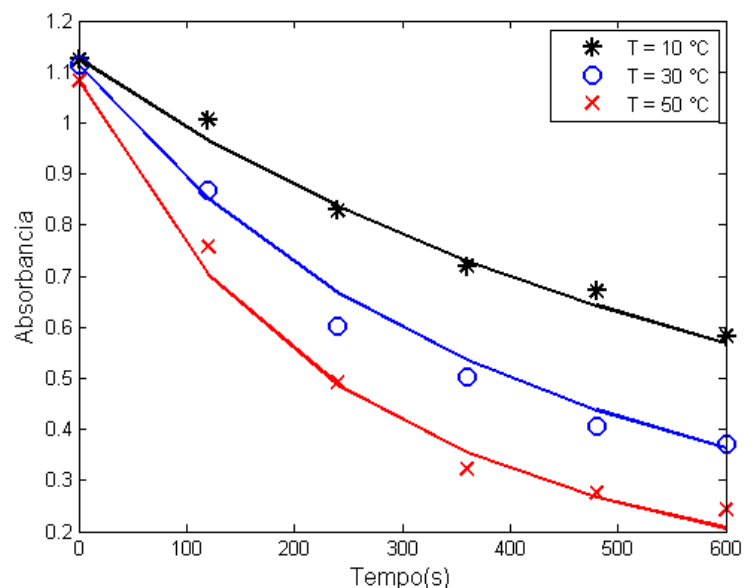

(a)

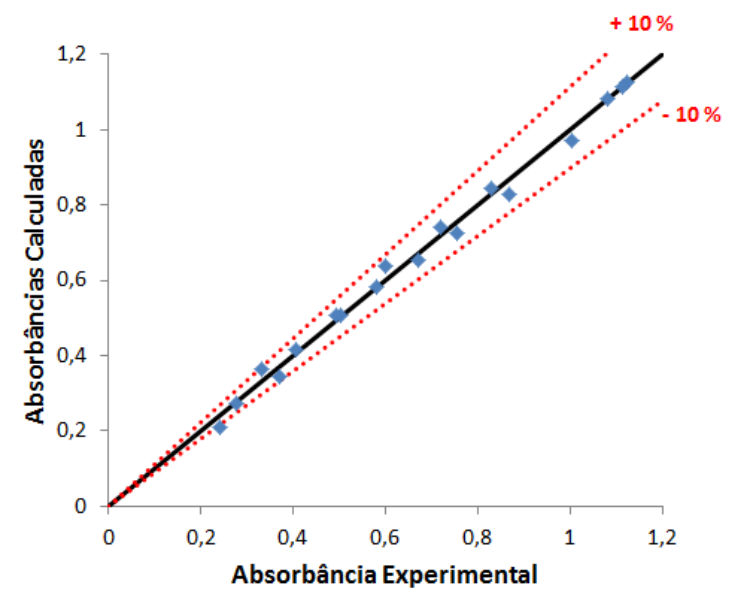

(b)

Figura 2 - (a) Pontos experimentais de Absorbância vs Tempo e curvas ajustadas individualmente para cada temperatura distinta. (b) Absorbâncias experimentais vsAbsorbâncias calculadas individualmente para cada temperatura.

O erro médio global entre os valores de absorbâncias calculados através do modelo matemático e as absorbâncias obtidas experimentalmente foi determinado de acordo com a equação 14.

$$
\text { Erro Médio Global }=\frac{1}{N} \sum_{1}^{N} \frac{\left|A b s_{c}(n)-A b s_{e}(n)\right|}{A b s_{e}(n)} * 100 \%
$$

em que: $\mathrm{N}=$ Número total de amostras $(\mathrm{N}=18) ; A b s_{c}(n)=$ Absorbância calculada pelo modelo matemático para a amostra $\mathrm{n} ; A b s_{e}(n)=$ Absorbância obtida experimentalmente para a amostra $n$.

Na Figura 2b é possível verificar que os erros entre os valores de absorbância calculados pelo modelo e os obtidos experimentalmente estão, em sua grande maioria, dentro da faixa de $\pm 10 \%$. O erro máximo foi de $14,13 \%$ e o erro médio para o conjunto de pontos ajustados 
individualmente de acordo com cada uma das três diferentes temperaturas foi de $3,62 \%$.

\subsection{GENERALIZAÇÃO DO MODELO}

A generalização do modelo matemático consiste em ajustar um único conjunto de coeficientes para a equação modelo de modo que tal equação seja válida independente da temperatura do caldo de cana-de-açúcar que estiver sendo utilizado. O conjunto de coeficientes genéricos foi obtido conforme descrito a seguir.

O coeficiente $\alpha$, que representa a ordem da reação de degradação dos pigmentos,apresentou valores próximos para as três temperaturas. Assim sendo, foi considerado constante assumindo o valor médio de $\alpha=1,4$.

O coeficiente $k_{a}$ representa a constante de proporção entre absorbância medida no caldo e a quantidade de pigmentos que dão cor ao caldo e apresentou, também, valores muito próximos para as três temperaturas quando $\alpha=1,4$. Portanto, seu valor também foi considerado constante assumindo o valor médio de $k_{a}=0,0038$.

Já os coeficientes $k_{p}$ e $k_{o}$ foram ajustados como um único coeficiente $k^{\prime}$ através da equação de Arrhenius:

$$
\ln k^{\prime}=\ln C a-\frac{E a}{R T}
$$

em que: $k=$ Constante de velocidade $\mathrm{Ca}=$ Constante de Arrhenius ou fator pré-exponencial; $E a=$ Energia de ativação; $R=$ Constante dos gases $\left(8,314 \mathrm{~J} \cdot \mathrm{K}^{-1} \cdot \mathrm{mol}^{-1}\right)$; $T=$ Temperatura absoluta em Kelvin.

Utilizando-se os coeficientes $k_{10}^{\prime}=0,0112, k_{30}^{\prime}=0,0227$ e $k_{50}^{\prime}=0,0338$ obtidos para as curvas ajustadas pelo script MATLAB para $\alpha=1,4$ e $k_{a}=0,0038$ traçou-se, com o auxílio do software Microsoft Excel, um gráfico de $\operatorname{lnk} k^{\prime} v s$ 1/T apresentado na Figura 3.

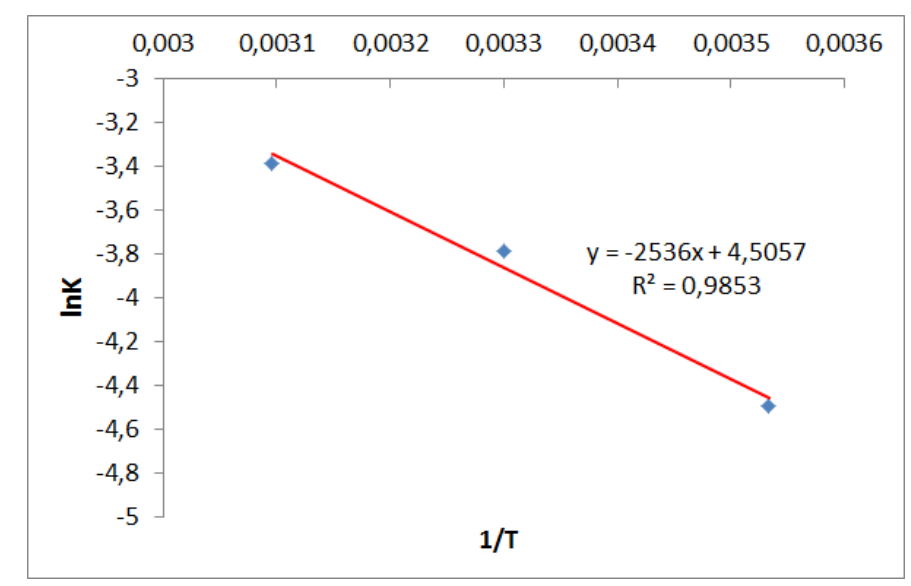

Figura 3 - Determinação do Coeficiente de Arrhenius e Energia de Ativação 
Dessa forma, o coeficiente linear da reta ajustada pelo Microsoft Excel representa $\ln C a$ e o coeficiente angular representa $-E a / R$. Assim, obteve-se o Coeficiente de Arrhenius $C a=$ 90,53 e a Energia de Ativação $E a=21084,3$.

Dessa forma, o modelo matemático generalizado para a clarificação do caldo de cana mediante ozonização em reator batelada ficou:

$$
\begin{aligned}
& A=\left(A_{0}^{1-\alpha}-C a e^{-E a / R T} k_{a}^{\alpha-1}(1-\alpha)\left(t-t_{0}\right)\right)^{1 /(1-\alpha)} \\
& \text { em que: } C a=90,53 ; E a=21084,3 ; k a=0,0038 ; \alpha=1,4 .
\end{aligned}
$$

As curvas obtidas com o modelo matemático generalizado podem ser vistas na Figura 4a.As absorbâncias experimentais e as absorbâncias obtidas pelo modelo generalizado podem ser vistos na Figura $4 \mathrm{~b}$.

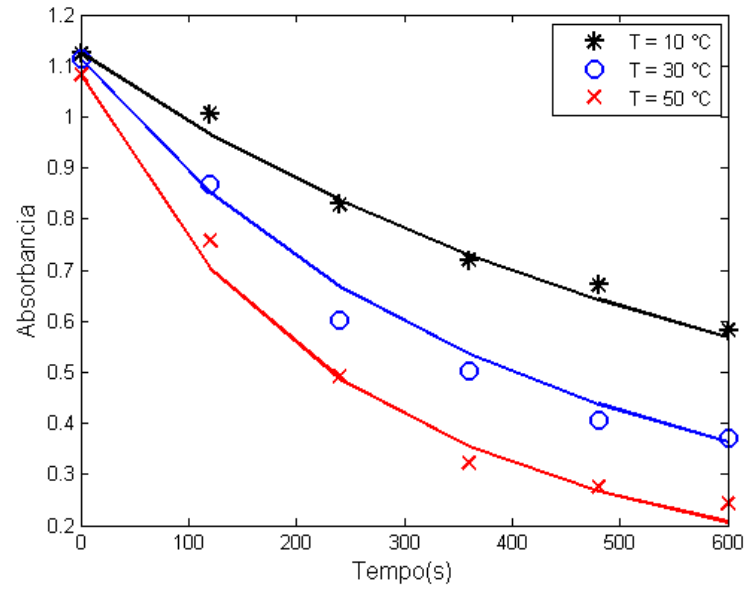

(a)

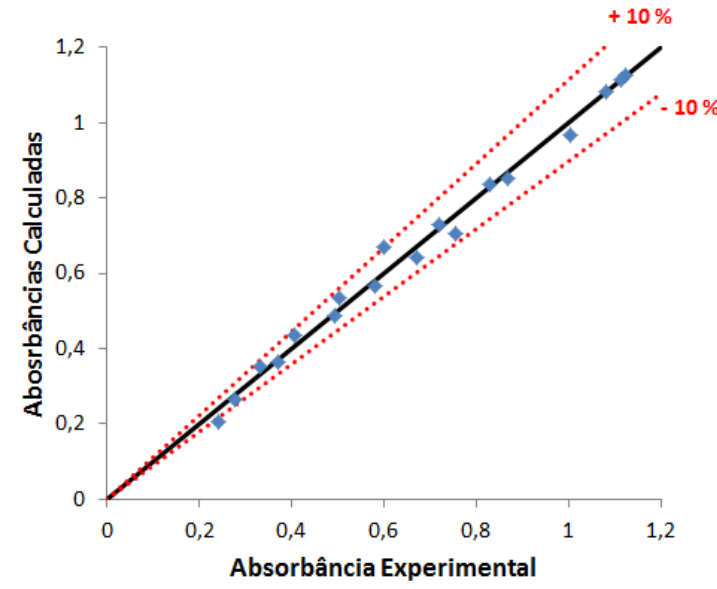

(b)

Figura 4 - (a) Pontos obtidos experimentalmente e curvas ajustadas pelo modelo matemático generalizado. (b) Absorbâncias experimentais vsAbsorbâncias calculadas pelo modelo matemático generalizado.

Pela Figura $4 b$ podemos verificar que os erros entre os valores calculados e os obtidos experimentalmente continuaram, em sua grande maioria, dentro da faixa de $\pm 10 \%$. O erro máximo foi de $14,62 \%$ e o erro médio global, calculado após a generalização do modelo, foi de 4,20\%.

\section{CONCLUSÕES}

Analisando as Figuras $2 \mathrm{~b}$ e $4 \mathrm{~b}$ podemos verificar que os erros individuais, para os dois casos, mantiveram-se, em sua grande maioria, dentro da faixa de $\pm 10 \%$ delimitados pelas linhas pontilhadas. Os erros, tanto máximos quanto médios globais, obtidos pelo conjunto de dados ajustados individualmente foram menores em relação aos erros obtidos pelo conjunto de dados ajustados pelo modelo generalizado. Dessa forma, pode-se perceber que a praticidade de se trabalhar com um único modelo ao invés de três modelos diferentes foi 
trocada por acréscimos nos erros obtidos. Contudo, tais acréscimos não foramconsiderados tão expressivos, permanecendo o erro médio global,após generalização do modelo, ainda abaixo dos $5 \%$.

Com base nos erros apresentado, conclui-se que o modelo matemático generalizado apresentado neste artigo representa bem a cinética de clarificação do caldo de cana-de-açúcar mediante ozonização em reator batelada, ao menos para as temperaturas estudadas.

\section{REFERÊNCIAS BIBLIOGRÁFICAS}

ARAÚJO, F. A. D. Processo de clarificação do caldo decana pelo método da bicarbonatação. Revista Ciências \& Tecnologia. Julho-Dezembro/2007.

ARMOA, M. H. Clarificação de caldo de cana-de-açúcar por filtração tangencial em membrana compósita titânia/sílica. Ciência \& Tecnologia. Jaboticabal, 2012.

LIMA, R. B. Processo de clarificação de caldo de cana-de-açúcar aplicando elétrons acelerados. Dissertação (Mestrado em Ciências) - Instituto de Pesquisas Energéticas e Nucleares - Universidade de São Paulo. São Paulo, 2012.

FOGLER, H. S. Elementos de Engenharia das Reações Químicas. Rio de Janeiro: LTC, 2008.

HAMERSKI, F. Estudo de variáveis no processo de carbonatação do caldo de canade-açúcar. Dissertação (Mestrado em Tecnologia de Alimento) - Universidade Federal do Paraná, Curitiba, 2009

RIBEIRO, P. Proposta de Projeto de Lei - PL-6639/09, 2009. Disponível em: http://www.camara.gov.br/proposicoesWeb/fichadetramitacao?idProposicao=464804. Acessado em: Fevereiro/2014.

SCHIAVON, G. J.Modelagem, desenvolvimento e análise de um sistema gerador de ozônio, operando em alta frequência com controlador digital de sinais. 265 p.Tese (Doutorado em Engenharia Química) - Programa de Pós-graduação em Engenharia Química - Universidade Estadual de Maringá. Maringá, 2012.

SILTON, R. N. C. Processo de clarificação do caldo de cana-de-açúcar por ozonização. Patente do Brasil PI 0505003-0 A,2007.

SILVA, R. B. da. Et al. Aplicação da produção mais limpa no processo de clarificação do caldo de cana para produção de açúcar. Encontro Nacional de Eng. de Produção. Rio de Janeiro, 2008.

HUNTERLAB, Specialty Color ScalesandIndices. Application Notes V. 17No. 10 / 2008. Disponível em: http://www.hunterlab.se/wp-content/uploads/2012/11/SCAA-Coffee-ColorStandards.pdf. Acessado em: Abril/2014. 